\title{
Improving the Hydromechanical Deep-Drawing Process Using Aluminum Tailored Heat Treated Blanks
}

\author{
Antonio Piccininni ${ }^{1} \cdot$ Gabriella Di Michele $^{1} \cdot$ Gianfranco Palumbo $^{1} \cdot$ Donato Sorgente $^{1} \cdot$ Luigi Tricarico $^{1}$
}

Received: 30 June 2015/Revised: 17 September 2015/Published online: 9 December 2015

(C) The Chinese Society for Metals and Springer-Verlag Berlin Heidelberg 2015

\begin{abstract}
The present work demonstrates the effectiveness of combining the hydromechanical deep-drawing process with the Tailored Heat Treated Blank (THTB) technique. In the hydromechanical deep-drawing process, the fluid pressure is used for postponing the fracture occurrence in the blank, while the THTB technique allows to create a material property gradient through a suitable artificial aging treatment carried out prior to the forming process. Since the number of process variables is large, in the present work the authors propose an optimization loop for the determination of the parameters controlling the extension of the blank regions to be subjected to the aging treatment and the temperature levels to be set during the heat treatment. The proposed methodology couples a simple finite element model (Abaqus) with a multiobjective optimization platform (modeFRONTIER). A preliminary experimental campaign was carried out for determining the effect of the aging treatment on the mechanical (through tensile tests) and deformative (through formability tests) behavior of the AC170PX aluminum alloy. Optimization results prove the effectiveness of the adopted methodology and put in evidence that the adoption of properly aged blanks in the hydromechanical deep drawing allows to increase the limit drawing ratio and to simplify the process since it is conducted at room temperature.
\end{abstract}

KEY WORDS: Aluminum alloy; Aging; Hydromechanical deep drawing; Finite element analysis; Mechanical characterization; Formability

\section{Introduction}

In recent years, the stricter normative framework for the pollutant emissions is pushing toward a massive adoption of light materials, in particular aluminum ( $\mathrm{Al}$ ) and magnesium $(\mathrm{Mg})$ alloys. In fact, such materials are characterized by good mechanical properties and low density (that is, high strength-to-weight ratios) [1]. The above-mentioned advantages are partially counterbalanced by the

Available online at http://link.springer.com/journal/40195

Gianfranco Palumbo

gianfranco.palumbo@poliba.it

1 Dipartimento di Meccanica, Matematica e Management, Politecnico di Bari, Viale Japigia 182, 70126 Bari, Italy well-known drawback of the poor formability at room temperature, which usually makes the manufacturing more complicated, thus leading to increase the number of forming steps. Such a limitation has been overcome in industrial applications by using: (1) more suitable forming processes, such as the hydroforming (HF) or the hydromechanical deep drawing (HDD); (2) higher working temperatures (in order to increase the value of the strain rate sensitivity [2] or to activate additional slip systems, like in $\mathrm{Mg}$ alloys [3]).

In the HF process, the action of the forming tool is completely replaced by pressurized oil, thus allowing to manufacture complex-shaped automotive parts [4] or bipolar plates for fuel cells [5], without the need of changing the tool if blanks with different thickness have to be used; in addition, rapid prototyping techniques can be also adopted to create the forming tools [6]. In the HDD, a 
fluid counter pressure is used for improving the conventional stamping process: For example, rectangular shape components can be more easily manufactured, without the need of intermediate stress relief heating steps [7]; in addition, a better control of the component thickness distribution can be achieved [8], and the risk of undesired defects, such as wrinkles and bursting, can be noticeably reduced [9], especially if the fluid pressure effect is combined with a suitable blankholder force level [10] and the proper initial geometry of the blank [11]. The adoption of a counter pressure was proved to increase the limit drawing ratio (LDR) due to: (1) the tangential stress along the wall of the cup due to the friction between the punch and the specimen; (2) the fluid lubrication effect, which reduces the friction between the flange of the specimen and the die. In addition, lower tooling cost, higher dimensional accuracy, less springback, shorter manufacturing cycle and better surface quality are additional advantages coming from the adoption of the counter pressure. The HDD process can be further improved if a pre-bulging is adopted [12] and/or if shallow drawbeads are created in the die tool for locally work-hardening the blank before the drawing [13].

The second strategy to overcome the problem of the poor formability which characterizes $\mathrm{Mg}$ and $\mathrm{Al}$ alloys is to increase the working temperature, usually creating a local heating/cooling of the blank during forming. For example, in the warm deep drawing (WDD) the thermal gradient between the peripheral region of the blank (the flange) and the central region of the blank (the one in contact with the punch) creates a suitable distribution of the mechanical properties able to counterbalance the stress gradient originated by the drawing. In the case of the Al alloy AA5754, large improvements of the LDR can be obtained maintaining the flange zone at high temperature while cooling the punch [14]. Investigations carried out on the $\mathrm{Mg}$ alloy AZ31 reported that, besides the LDR improvement due to the thermal gradient [15], further beneficial effects can be obtained decreasing the punch speed (or the strain rate the alloy is subjected to) [16].

An approach similar to the adoption of the local heating/cooling is the tailoring of the blank properties; the Tailored Heat Treated Blank (THTB) technology, widely adopted in the automotive field for the age-hardenable $\mathrm{Al}$ alloys, is based on local aging treatments prior to the stamping aimed to improve the material drawing or the material strength, thus creating, also in this case, an optimal distribution of the material properties able to counterbalance the stress gradient originated by the sheetforming process. In such a way, the stamping process can be performed at room temperature, with consequent less expensive equipment and tools [17].

Although the above-mentioned technologies clearly prove the feasibility of enhancing the process performance, few results are present in the literature regarding the advantages coming from the adoption of more than one approach simultaneously. The present work deals with the numerical investigation of the HDD process at room temperature using an age-hardenable $\mathrm{Al}$ alloy sheet which was heat treated prior to the forming process (THTB technique) in order to determine an optimal distribution of the mechanical properties. Experiments and simulations proposed in this work were carried out on a $6 \mathrm{xxx}$ series $\mathrm{Al}$ alloy (AC $170 \mathrm{PX}$ ). Optimal values of both aging temperatures and extension of the blank regions to be heat treated were numerically determined coupling a numerical model created with the commercial finite element (FE) code Abaqus (v. 6.12) within the integration platform modeFRONTIER (v. 4.5.3). Results of the optimization demonstrate the effectiveness of the proposed approach in obtaining an LDR higher than those actually reported in the literature for similar Al alloys.

\section{Alloy Characterization}

The investigated alloy is the age-hardenable Al alloy AC $170 \mathrm{PX}$, belonging to the $6 \mathrm{xxx}$ series. It is purchased in the T4 condition, and its chemical composition is reported in Table 1.

Tensile tests were performed at room temperature on previously aged specimens using the experimental setup shown in Fig. 1a: a $200 \mathrm{kN}$ electromechanical INSTRON tensile test machine was used in conjunction with a digital image correlation (DIC) system (Aramis, GOM); to correlate image series, aged specimens were painted to obtain a stochastic pattern (Fig. 1b) so that the DIC system could calculate the complete strain field distribution over the specimen.

The crosshead speed was set to $6 \mathrm{~mm} / \mathrm{min}$. Before the test, specimens were subjected to different aging temperatures (in the range $150-400{ }^{\circ} \mathrm{C}$, with step of $50{ }^{\circ} \mathrm{C}$ ) for different aging times (12, 600 and $1200 \mathrm{~s}$ at each temperature) in order to evaluate the effect of the aging parameters on the plastic behavior of the alloy.

Specimens were cut (by water jet) along the rolling direction. A summary of results from tensile tests is reported in Fig. 2, where the YS\% is the yield stress referred to the correspondent value in the as-received condition and the UTS\% is the ultimate tensile strength referred to the correspondent value in the as-received condition.

Table 1 Chemical composition of AC170PX alloy (in wt \%)

\begin{tabular}{lllllllll}
\hline $\mathrm{Si}$ & $\mathrm{Fe}$ & $\mathrm{Cu}$ & $\mathrm{Mn}$ & $\mathrm{Mg}$ & $\mathrm{Cr}$ & $\mathrm{Zn}$ & $\mathrm{Ti}$ & $\mathrm{Al}$ \\
\hline 0.6 & 0.16 & 0.14 & 0.06 & 0.7 & 0.0 & 0.0 & 0.0 & Bal. \\
\hline
\end{tabular}



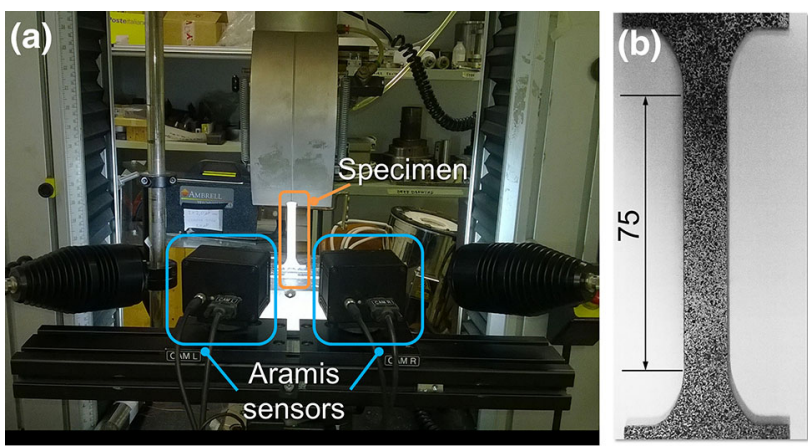

Fig. 1 a Experimental setup for tensile tests on aged specimens; b stochastic pattern created on the specimen surface (unit: $\mathrm{mm}$ )

According to the proposed plots, the lowest investigated aging time (12 s) has a negligible effect on the mechanical properties of the alloy; on the other hand, when focusing the attention on the aging time of $600 \mathrm{~s}$, it can be noted that the YS\% reaches its maximum values in the range between 250 and $300{ }^{\circ} \mathrm{C}$ (peak hardening), while temperature values higher than $300{ }^{\circ} \mathrm{C}$ lead to a reduction in the YS\% even below that of the as-received material due to overaging. Although changes are more moderate, the UTS\% values show a similar trend to the YS\% at all aging conditions investigated. The above-described results are in agreement with the mechanical properties after aging reported in Ref. [18] for a similar alloy (AA6181PX).

In order to investigate the deformative behavior of the alloy, formability tests were carried out. The experimental setup for Nakajima tests, assembled on the same machine used for tensile tests, is shown Fig. 3a: It is composed of a hemispherical punch having a diameter of $90 \mathrm{~mm}$, a circular draw die and a blank holder ring; in addition, the DIC system was embedded in the equipment to acquire the strain field during the test.

Formability tests were performed at room temperature on specimens previously heat treated, setting different aging temperatures $\left(200,300\right.$ and $\left.400{ }^{\circ} \mathrm{C}\right)$ but keeping the aging time unchanged $(600 \mathrm{~s})$. Results have been resumed in Fig. 3b in terms of forming limit curves (FLCs). It can be noted that if the aging temperature is reduced, the FLC is shifted downward, thus confirming the effect of the secondary phase precipitation in limiting the alloy formability.

\section{Finite Element Model}

In order to minimize the computational costs of the optimization loop detailed in the following section, a simple and low time-consuming axisymmetric FE model (Abaqus v. 6.12) was created and used for the numerical analyses. The material anisotropy was neglected in the present investigation, and the isotropic Von Mises criterion was used for modeling the material yield behavior. On the contrary, the material behavior was accurately modeled implementing flow stress data obtained by tensile tests on specimens previously subjected to different aging treatments (aging temperatures ranging from 200 and $400{ }^{\circ} \mathrm{C}$, while aging time kept constant at the value of $600 \mathrm{~s}$ ).

Figure 4a shows the FE model: the punch, the blankholder and the die were modeled as analytical rigid parts, whereas the blank as a deformable one (initial thickness equal to $1 \mathrm{~mm}$ ) adopting SAX axisymmetric shell elements (size equal to $0.25 \mathrm{~mm}$ ) having a high enough number of through-thickness integration points able to guarantee the accuracy of results without excessively increasing the computational cost.

During the simulation, the fluid pressure in the forming chamber and the punch stroke grew according to the linear profiles shown in Fig. 4b. It is important to note that the punch started its stroke when the pressure under the blank reached a predefined level (pre-bulging pressure) of $10 \mathrm{MPa}$ (kept unchanged for all the simulations).
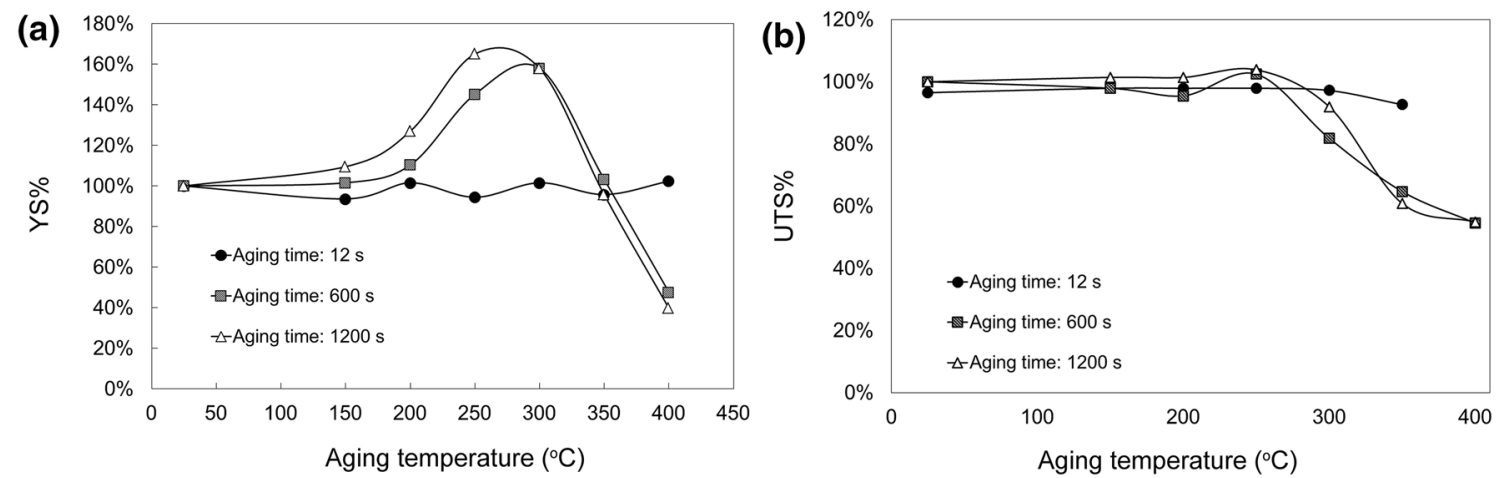

Fig. 2 Summary of results from the mechanical characterization of aged specimens in terms of percentage yield stress (YS\%) a and percentage ultimate tensile strength (UTS\%) b 

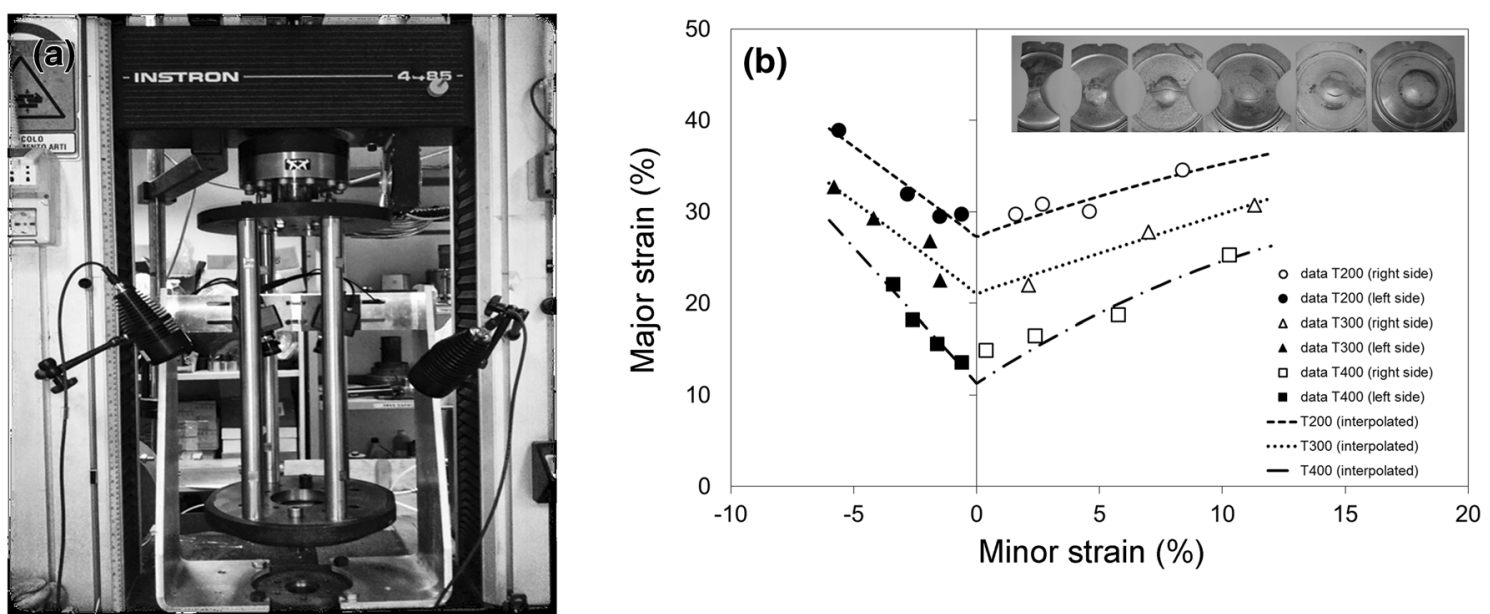

Fig. 3 Formability test: a equipment; b FLCs concerning specimens subjected to different aging temperatures
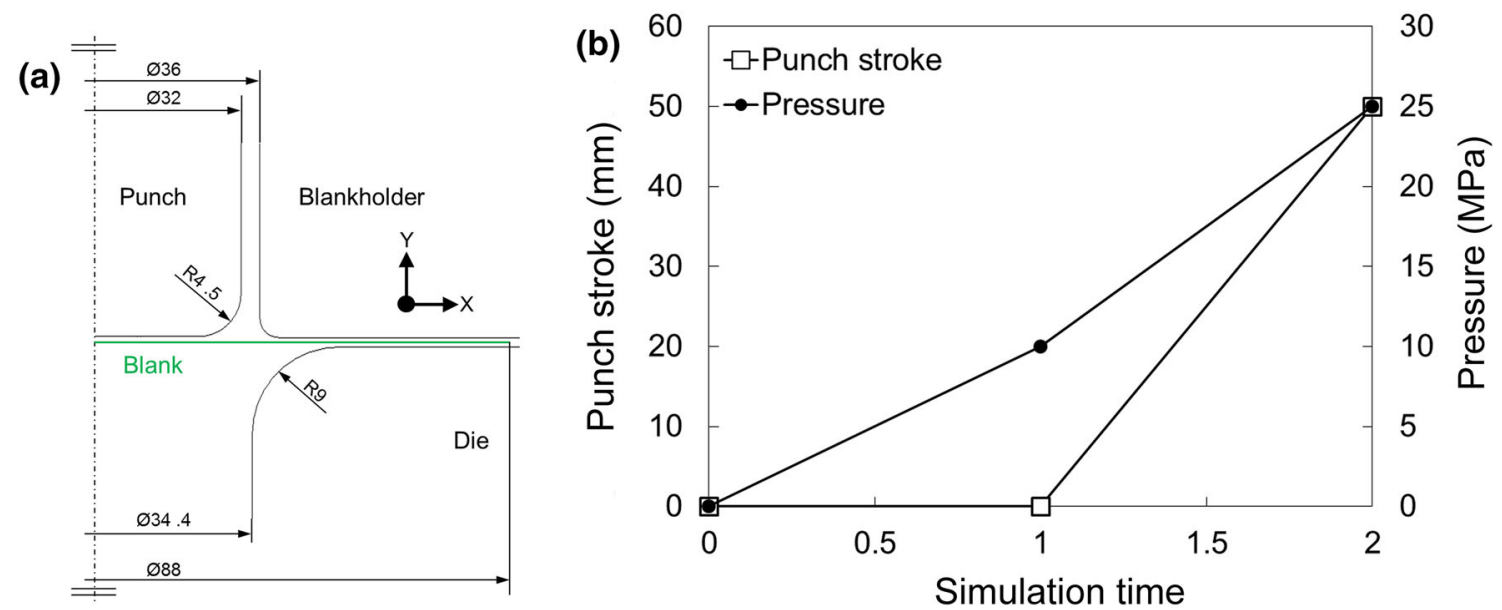

Fig. 4 a FE model; $\mathbf{b}$ adopted pressure and punch stroke profiles

Two different values of the coefficient of friction $(\mathrm{CoF})$ were adopted in the numerical model: similarly to what proposed by Halkaci [13], the CoF modeling the interactions (contact pairs) die/blank and blankholder/blank was set equal to 0.05 , while the $\mathrm{CoF}$ modeling the interaction punch/die was set equal to 0.2 .

In the numerical simulations, mechanical properties of the alloy were locally set by changing the temperature level over the blank. In the proposed HDD process, the working temperature is the room one but, in order to easily change material properties in the optimization loop, predefined temperature fields were used in the numerical model. In particular, both the level of the aging temperatures and the extension of the blank regions subjected to the aging treatment prior to the HDD process were taken into account. According to Fig. 5a, the parameter $L_{1}$ was used to define the extension of the central region of the blank (the one in contact with the punch) subjected to the aging temperature $T_{1}$. In a similar way, the parameters $L_{2}$ and $T_{2}$ were used to define the extension and the aging temperature in the flange region, respectively. In order to make the simulation as close as possible to the real process without losing the simplicity of the adopted model, the portion of the blank between the two regions having the extensions $L_{1}$ and $L_{2}$ was assumed to be subjected to an intermediate aging temperature (i.e., the aging temperature in the abovementioned portion of the blank was calculated as the arithmetic mean of $T_{1}$ and $T_{2}$ ). For example, the temperature map in Fig. 5a concerns the simulation carried out setting $T_{1}$ to $200{ }^{\circ} \mathrm{C}$ and $T_{2}$ to $400{ }^{\circ} \mathrm{C}$.

As put in evidence, the sharp discontinuity between the temperatures in the regions $L_{1}$ and $L_{2}$ and the temperature in the middle portion $\left(300{ }^{\circ} \mathrm{C}\right)$ was avoided creating two transition regions (extension is $3 \mathrm{~mm}$ ).

Numerical results were collected taking into account two output parameters: (1) the maximum punch stroke 


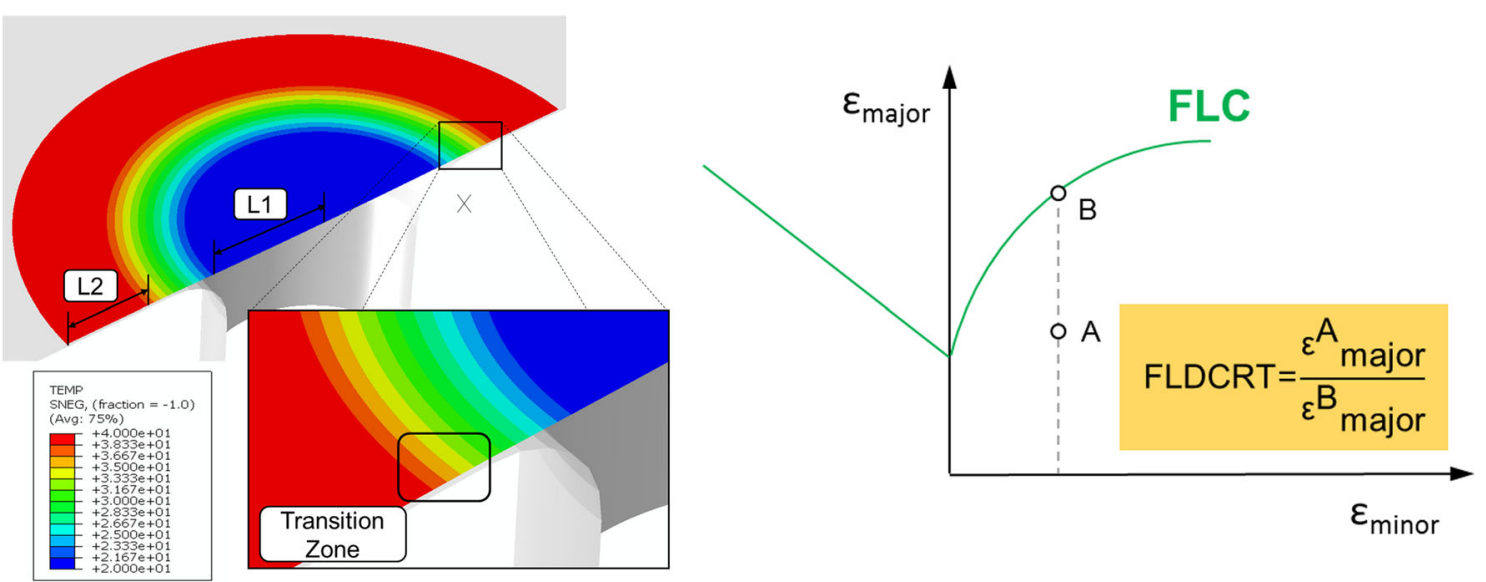

Fig. 5 a Temperature map superimposed to the blank for simulating the behavior of the THTB; $\mathbf{b}$ definition of the parameter FLDCRT

prior to fracture; (2) the FLDCRT (calculated, according to Fig. $5 b$, as the ratio between the actual value of the nodal major strain and the FLC limit major strain at the same level of nodal minor strain) in the blank region located in front of the punch fillet radius.

\section{Optimization Procedure}

The FE model was coupled within the integration platform modeFRONTIER in order to create a multi-objective genetic algorithm optimization procedure. The optimization loop had the ultimate goal of determining the values of the above-defined parameters $\left(L_{1}, T_{1}, L_{2}\right.$ and $\left.T_{2}\right)$ able to optimize the process for a blank having the diameter of $88 \mathrm{~mm}$. The ranges of variation of the input parameters are reported in Table 2 .

It can be noted that the range of variation for $T_{1}$ and $T_{2}$ is the same adopted in the mechanical characterization, while the ranges for $L_{1}$ and $L_{2}$ were determined under the following hypotheses: (1) to ensure a minimal heat-treated region under the punch $\left(L_{1}=10 \mathrm{~mm}\right.$ at least) and under the blankholder ( $L_{2}=10 \mathrm{~mm}$ at least); (2) to extend the heat-treated region up to the end of the fillet radius of the punch (for $L_{1}$ ) and up to the end of the die (for $L_{2}$ ).

The flowchart in Fig. 6 clarifies how the optimization procedure works: the optimal combination of the input variables (the vector made by the parameters $L_{1}, T_{1}, L_{2}, T_{2}$ )

Table 2 Ranges of variation of the adopted input parameters

\begin{tabular}{lcc}
\hline Input variable & Lower value & Upper value \\
\hline$L_{1}(\mathrm{~mm})$ & 10 & 19 \\
$L_{2}(\mathrm{~mm})$ & 10 & 14 \\
$T_{1}\left({ }^{\circ} \mathrm{C}\right)$ & 200 & 400 \\
$T_{2}\left({ }^{\circ} \mathrm{C}\right)$ & 200 & 400 \\
\hline
\end{tabular}

is determined looking for a vector able to both maximize the Punch Stroke and minimize the FLDCRT level in the fillet radius region.

The following constraint was applied to the output variable punch stroke: runs in which rupture (FLDCRT $>1$ ) occurred before reaching the maximum punch stroke (set to $50 \mathrm{~mm}$ in all simulations) were not considered by the genetic algorithm for the creation of successive generations. An initial population of 50 designs was created by the Sobol algorithm. As shown in Fig. 7, such an algorithm is able to uniformly distribute the population within the design space avoiding the risk of clusters [18].

Starting from the initial population, the optimization loop then evolved according to the flowchart in Fig. 6. In particular, successive generations were limited to 20; each generation was created by the genetic algorithm (MOGAII) adopting the mutation and the crossover operators [19].

\section{Results and Discussion}

\subsection{Effect of the Aging Temperature}

The way the optimization loop evolved toward the optimal values of the two input variables related to the aging

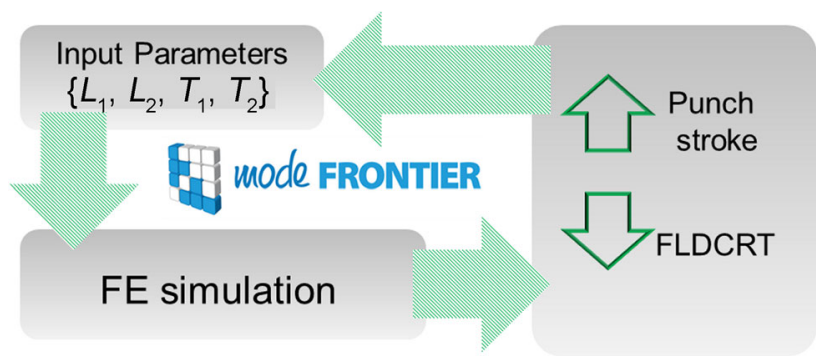

Fig. 6 Flowchart of the optimization loop 

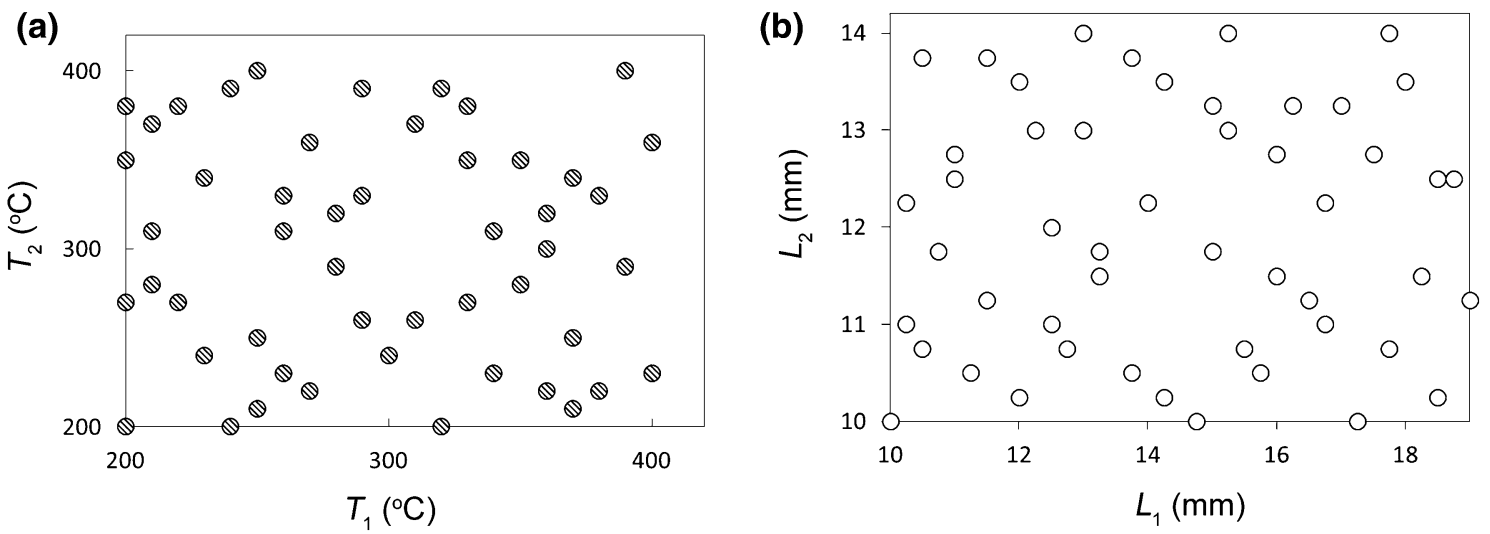

Fig. 7 Distribution of the initial population within the design space for the parameters: a $T_{1}$ and $T_{2} ; \mathbf{b} L_{1}$ and $L_{2}$
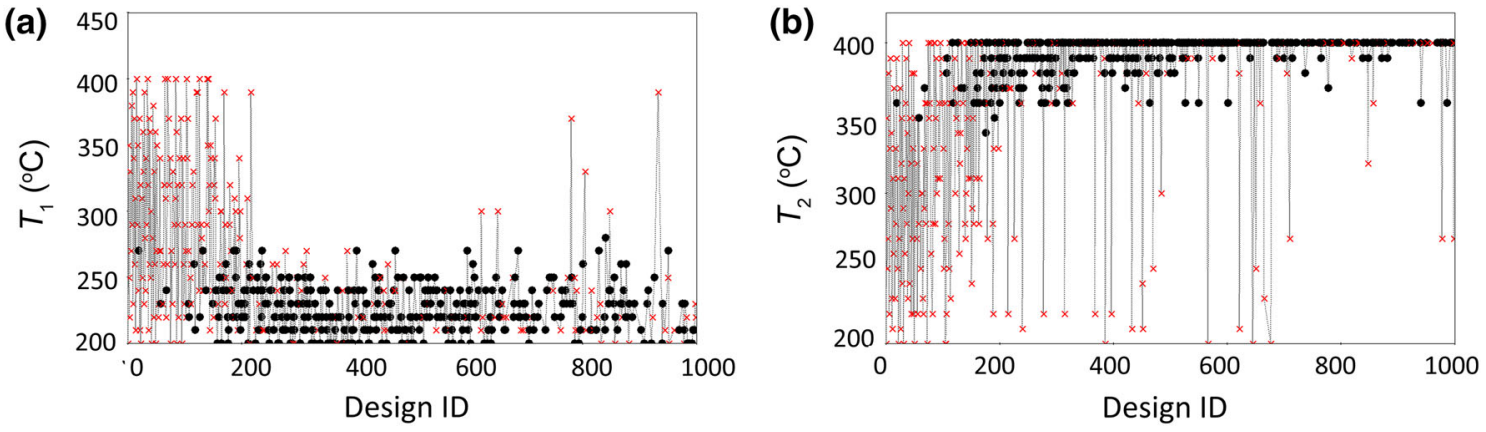

Fig. 8 History charts of the two input variables related to the aging temperatures $T_{1} \mathbf{a}$ and $T_{2} \mathbf{b}$

temperature $\left(T_{1}\right.$ and $\left.T_{2}\right)$ is resumed in Fig. 8 by means of history charts.

In the proposed plots, each dot refers to a feasible (i.e. able to respect the applied constraint on the punch stroke) design, being each design a vector composed of the four input variables; on the contrary, each cross refers to an unfeasible design. From the analysis of the plot in Fig. 8a, it is easy to note that sound cups can be obtained only by heat treating the central blank region (the one in contact with the punch) up to a temperature level in the range of 200-250 ${ }^{\circ} \mathrm{C}$; at the same time, according to Fig. 8 b, in the peripheral region of the blank (the one in contact with the blankholder) it is necessary to perform the aging treatment, setting the aging temperature to $400{ }^{\circ} \mathrm{C}$.

Results from history charts have been also confirmed by the contour plot in Fig. 9, which concerns the response surface created fitting data of the output variable punch stroke by means of an interpolating radial basis function algorithm [20].

The contour plot highlights that sound and well-formed specimens (i.e. the one where punch stroke values are the highest) can be obtained if the aging parameters $T_{1}$ and $T_{2}$ fall in the upper left corner region of the diagram, which is characterized by a relatively large range for the parameter $T_{1}$ but quite a sharp value for the parameter $T_{2}$.

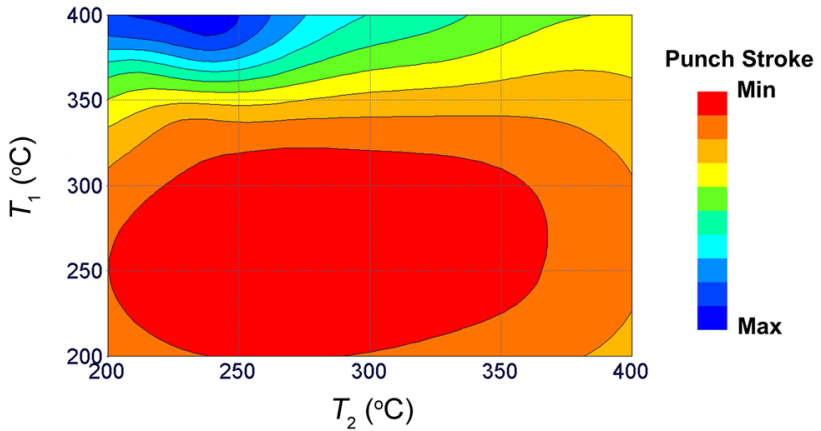

Fig. 9 Contour plot of the output variable punch stroke

\subsection{Effect of the Extension of the Aged Regions}

History charts have been also used for detailing the results of the optimization loop concerning the two input variables which control the extension of the aged regions $\left(L_{1}\right.$ and $\left.L_{2}\right)$.

Figure 10a clearly suggests that the optimal value for $L_{1}$ is quite close to the upper range limit (about $19 \mathrm{~mm}$ ). On the contrary, the parameter $L_{2}$ (Fig. 10b) appears not converging toward a unique value, but it oscillates in the whole range.

In order to better understand the effect of the parameter $L_{2}$ and to define also its optimal value, the three 
simulations listed in Table 3 were carried out. Such simulations were aimed at investigating exclusively the effect of the parameter $L_{2}$, while keeping the others unchanged and fixed to the optimal values determined by means of the optimization loop and previously detailed.

Figure 11 plots the evolutions along the radial direction of the parameters sheet thickness (STH) and FLDCRT. In addition, the portion of the blank located in front of the punch radius, usually the critical one in such a process, is highlighted in Fig. 11.

It is remarkable that fracture was always avoided, thus providing a possible reason why the optimization loop could not converge toward a unique value. In addition, the analysis of the FLDCRT plots also suggests that an increasing value of the $L_{2}$ parameter has the effect of making the strain condition less critical; the reduction of FLDCRT values is more evident if $L_{2}$ is increased from 10 to $12 \mathrm{~mm}$ than from 12 to $14 \mathrm{~mm}$.

As concerning the thickness distribution, at the transition point between regions characterized by different aging temperature (points A, B, C in the graph), a discontinuity in the STH evolution can be detected. But the value of the parameter $L_{2}$ affects the STH evolution in the whole wall region and not only in the transition point; in particular, it determines an increase of the thickness if larger $L_{2}$ values are adopted.

The values of the sheet thickness variation (STV), calculated as the difference between the maximum and minimum thickness values along the radial direction, have been reported in Fig. 12 along with the maximum value of the parameter FLDCRT.

It can be concluded that, when the extension $L_{2}$ is set to the intermediate value of the range $(12 \mathrm{~mm})$, although the severity of the strain is not minimal, a more uniform thickness distribution in the formed part can be obtained. The above-mentioned value can be thus included in the set of the optimal values of the input variables.

It is worthy of notice that the successful drawing of the initial blank diameter of $88 \mathrm{~mm}$ corresponds to an LDR
Table 3 List of numerical simulations to define the optimal value of the parameter $L_{2}$

\begin{tabular}{lllll}
\hline Run & $L_{1}(\mathrm{~mm})$ & $L_{2}(\mathrm{~mm})$ & $T_{1}\left({ }^{\circ} \mathrm{C}\right)$ & $T_{2}\left({ }^{\circ} \mathrm{C}\right)$ \\
\hline No. 1 & 19 & 10 & 200 & 400 \\
No. 2 & 19 & 14 & 200 & 400 \\
No. 3 & 19 & 12 & 200 & 400 \\
\hline
\end{tabular}

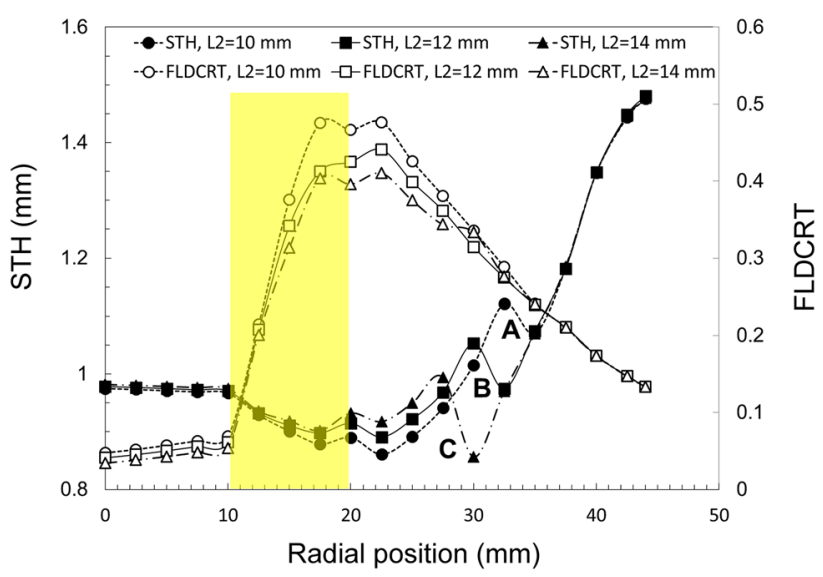

Fig. 11 Distribution of the parameters sheet thickness (STH) and FLDCRT along the radial direction

equal to 2.75: such an LDR value is higher than the ones predicted by numerical simulations carried out taking into account separately the counter pressure $(\mathrm{LDR}=2.375)$ and the aging treatment using optimal values for $L_{1}, T_{1}, L_{2}$ and $T_{2}(\mathrm{LDR}=2.5)$.

In addition, the achieved LDR is a further improvement if compared to experimental results available in the literature: a lower LDR (2.54) was obtained by Lang et al. [21] using the HDD process for forming an AA6016-T4 sheet, although the maximum pressure value was similar to the one used in the present work.

Moreover, if the warm deep drawing (characterized by a thermal gradient in the radial direction of the blank
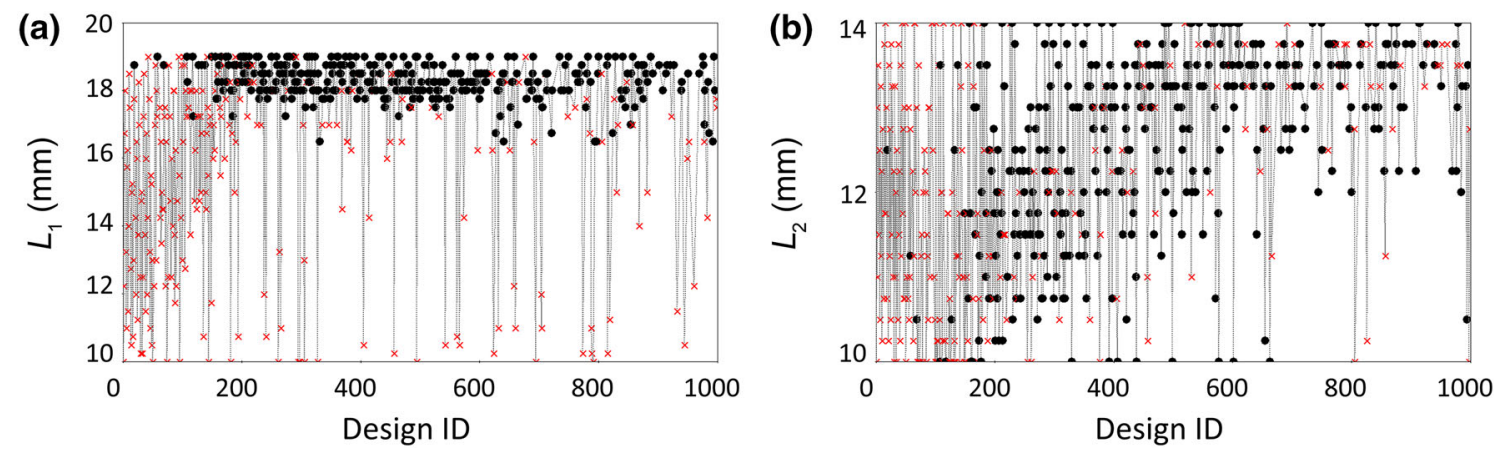

Fig. 10 History plots of the two input variables $L_{1}$ a and $L_{2} \mathbf{b}$ related to the extension of the aged regions 


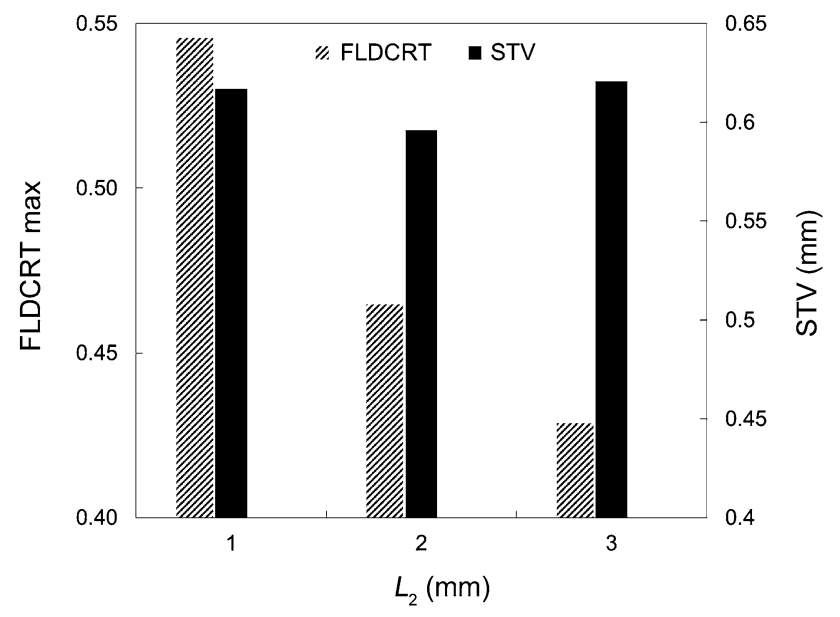

Fig. 12 STV and maximum FLDCRT value according to $L_{2}$

produced by heated/cooled tools) is considered as reference, the LDR found in the literature for the same investigated alloy (2.5 [22]) is lower than the one obtained in the present work and it is equal to the one predicted when adopting only the local aging treatment of the blank.

\section{Conclusions}

A numerical methodology is presented in this work to determine optimal values of the parameters controlling the artificial aging treatment carried out on blanks to be formed by hydromechanical deep drawing.

Starting from the knowledge of the effect of the aging treatment on the mechanical and deformative material behavior (obtained in the present work through tensile and formability tests on aged specimens), the proposed methodology couples a simple FE model with a multi-objective optimization platform.

The proposed optimization loop reveals to be effective in determining the temperature values to be used for the local aging treatments of the blank. On the contrary, the optimization loop is only able to determine the optimal extension of the central blank region $\left(L_{1}\right)$ to be aged; as concerns the extension of the peripheral blank region to be subjected to the heat treatment $\left(L_{2}\right)$, all values in the investigated range $(10-14 \mathrm{~mm})$ allowed to avoid fracture.

In particular, an LDR value equal to 2.75 is predicted when coupling the pressure action with the material property gradient determined by the aging treatment performed setting the temperature in the central region of the blank to a value in the range $200-250{ }^{\circ} \mathrm{C}$ and the temperature in the peripheral region of the blank to $400{ }^{\circ} \mathrm{C}$.

The numerically achieved value of LDR (2.75) is higher than the one obtained on similar alloys in the literature. In fact, numerical simulations carried out keeping the two effects (the oil pressure and the aging treatment) separated predicted an LDR value of 2.375 and 2.5 , respectively. A similar LDR (2.5) is obtained in the literature by the warm deep-drawing process, thus using heated/cooled tools able to determine a suitable thermal gradient in the blank during the forming process. On the contrary, the adoption of properly aged blanks in the hydromechanical deep drawing allows to increase the limit drawing ratio and to simplify the process since it is conducted at room temperature.

Acknowledgments The authors wish to thank Region APULIA and the Ministry of Education, University and Research for financing equipment and facilities adopted for the present research activity (Projects Acronyms: TRASFORMA, code 028, and SMATI, Grant Number PON01_02584).

\section{References}

[1] J. Hirsch, Trans. Nonferrous Met. Soc. China 24, 1995 (2014)

[2] D. Li, A. Ghosh, Mater. Sci. Eng. A 352, 279 (2003)

[3] L. Wang, Q. Qiao, Y. Liu, X. Song, J. Magn. Alloys 1, 312 (2013)

[4] M.H. Parsa, P. Darbandi, J. Mater. Process. Technol. 198, 381 (2008)

[5] G. Palumbo, A. Piccininni, Int. J. Adv. Manuf. Technol. 69, 731 (2013)

[6] G. Palumbo, Mater. Des. 44, 365 (2013)

[7] B. Meng, M. Wan, S. Yuan, X. Xu, J. Liu, Z. Huang, Int. J. Mech. Sci. 77, 217 (2013)

[8] K.S. Swadesh, D. Ravi, Kumar. J. Mater. Process. Technol. 204, 169 (2008)

[9] X. Liu, Y.C. Xu, S.J. Yuan, Trans. Nonferrous Met. Soc. China 21, 417 (2011)

[10] Y.J. Luo, Y.Q. Zhang, D.N. He, Acta Metall. Sin. (Engl. Lett.) 16, $31(2003)$

[11] L.H. Lang, D.C. Kang, S.H. Zhang, Z.R. Wang, S.J. Yuan, K.B. Nielsen, J. Dancket, Acta Metall. Sin. (Engl. Lett.) 13, 476 (2000)

[12] W. Liu, Y. Xu, S. Yuan, Procedia Eng. 81, 914 (2014)

[13] H.S. Halkaci, M. Turkoz, M. Dilmec, J. Mater. Process. Technol. 214, 1638 (2014)

[14] G. Palumbo, L. Tricarico, J. Mater. Process. Technol. 184, 115 (2007)

[15] G. Palumbo, D. Sorgente, L. Tricarico, S.H. Zhang, W.T. Zheng, J. Mater. Process. Technol. 191, 342 (2007)

[16] D. Ghaffari Tari, M.J. Worswick, S. Winkler, J. Mater. Process. Technol. 213, 1337 (2013)

[17] H. Nguyen, M. Merklein, Phys. Procedia 39, 318 (2012)

[18] modeFRONTIER, v. 4.5.3. User's Manual, Esteco

[19] K. Deb, Evol. Comput. 7, 205 (1999)

[20] M.D. Buhmann, D. Martin, Radial Basis Functions: Theory and Implementations (Cambridge University Press, UK, 2003), p. 99

[21] L. Lang, J. Danckert, K.B.K.B. Nielsen, Int. J. Mach. Tool Manuf. 44, 495 (2004)

[22] M. Ghosh, A. Miroux, R.J. Werkhoven, P.J. Bolt, L.A.I. Kestens, J. Mater. Process. Technol. 214, 756 (2014) 